\title{
A Trace-Based View on Operating Guidelines
}

\author{
Christian Stahl ${ }^{1}$ and Walter Vogler ${ }^{2}$ \\ 1 Department of Mathematics and Computer Science, \\ Technische Universiteit Eindhoven, The Netherlands \\ c.stahl@tue.nl \\ 2 Institut für Informatik, Universität Augsburg, Germany \\ vogler@informatik. uni-augsburg.de
}

\begin{abstract}
Operating guidelines have been introduced to characterize all controllers for a given service $S$. A controller of $S$ is a service that interacts with $S$ without deadlocking. An operating guideline of $S$ can be used to decide whether $S$ refines another service. It is a special-purpose structure to describe the behavior of service $S$ from the perspective of its controllers rather than from the perspective of $S$.

This paper provides a more conceptual understanding of operating guidelines from the perspective of a traditional concurrency semantics: a trace-based semantics. As benefits, we get an easier characterization of service refinement, and prove that this is a fully abstract precongruence.
\end{abstract}

\section{Introduction}

Service-oriented computing (SOC) 14 is a computing paradigm that has found growing acceptance in industry. A service-oriented system is a distributed system that is aggregated from building blocks, called services. A service encapsulates a certain functionality and offers it to other services over a well-defined interface. The distributed nature of service-oriented systems requires services to interact with each other via asynchronous message passing.

Service orientation supports the shift from monolithic systems to systems in which multiple organizations are involved, and it allows faster changes than in monolithic systems. The latter requires a notion of service replaceability. Replacing one service with another one has to preserve correctness of the overall system (compositionality). Because organizations usually do not know the services involved in the system, replacement must be independent of a service context; that is, for every context a service correctly interacts with, the replacement must correctly interact with as well. We refer to such a context as a controller.

A minimal requirement for the correctness of a system is the absence of deadlocks - that is, of unsuccessful termination. The replacement (or refinement) relation in the context of deadlock freedom has been formalized by the accordance preorder in [15]. The current decision procedure uses that, for finite-state services with bounded buffers, the set of controllers has a finite representation, the operating guideline 8] of the service; technically, it is an automaton annotated with Boolean formulae. Deciding accordance of two services is reduced to 
checking that one of the corresponding operating guidelines simulates the other and that the corresponding formulae of related states imply each other.

This characterization of accordance differs from existing refinement relations, because the special-purpose operating guideline describes the service behavior from the perspective of a controller and has an unusual nature. This makes operating guidelines more difficult to understand. In addition, it raises the question how the semantics of an operating guideline and hence the accordance preorder are related to existing refinement notions in literature. Another open question is whether accordance is a precongruence w.r.t. composition.

This paper answers these questions. First, we show that the behavior of a service can be characterized by four trace sets - somewhat similar to standard or completed traces, and to catastrophic traces in the spirit of divergence traces [2]. This semantics naturally gives rise to a refinement relation, which we prove to coincide with (a slight modification of) accordance. Like in 815, we use Petri nets to model finite state services; however, as a further improvement, we consider a more general notion of finite state service than in 815.9.

Second, our modified accordance relation is more uniform than the original accordance relation. In particular, the semantics of a composition can be determined from the semantics of the components, which proves that this relation is a precongruence w.r.t. composition. The latter can also be phrased as a full abstractness result. We further sketch how accordance can be decided in practice.

Third, we show explicitly how (automaton representations of) the new semantics can be translated back and forth into operating guidelines. This allows us to take a different view on operating guidelines.

Section 2 gives some background, Sect. 3 introduces an accordance relation for infinite state services and characterizes its semantics using traces. In Sect. 4, we present our results concerning accordance for finite state services. Section 5 compares operating guidelines and our trace-based semantics. We close with a discussion of related work and a conclusion.

\section{Preliminaries}

As a basic model, we use place/transition Petri nets extended with final markings and transition labels. For sets $A$ and $B, A \uplus B$ denotes the disjoint union; writing $A \uplus B$ expresses the implicit assumption that $A$ and $B$ are disjoint.

Definition 1 (net). A net $N=\left(P, T, F, m_{N}, \Omega\right)$ consists of a finite set $P$ of places, a finite set $T$ of transitions such that $P$ and $T$ are disjoint, a flow relation $F \subseteq(P \times T) \cup(T \times P)$, an initial marking $m_{N}$, where a marking is a mapping $m: P \rightarrow \mathbb{N}$, and a set $\Omega$ of final markings.

A labeled net is a net $N$ together with an alphabet $\Sigma$ of actions and a labeling function $l: T \rightarrow \Sigma \uplus\{\tau\}$, where $\tau$ represents an invisible, internal action.

Introducing $N$ implicitly introduces its components $P, T, F, m_{N}, \Omega$; the same applies to $N^{\prime}, N_{1}$, etc. and their components $P^{\prime}, T^{\prime}, \ldots$, and $P_{1}, T_{1}, \ldots$, resp. and it also applies to other structures later on. We use the standard graphical 
representation, writing transition labels $\neq \tau$ into the respective boxes. The preset of $x \in P \cup T$ is ${ }^{\bullet} x=\{y \mid(y, x) \in F\}$ and the postset is $x^{\bullet}=\{y \mid(x, y) \in F\}$. We interpret pre- and postsets as multisets when used in operations with multisets.

For a word $w \in \Sigma_{1}^{*}$ and $\Sigma_{2} \subseteq \Sigma_{1},\left.w\right|_{\Sigma_{2}}$ denotes the projection of $w$ to the subalphabet $\Sigma_{2}$. With $v \sqsubseteq w$ we denote that $v$ is a prefix of word $w$, and $\operatorname{cont}(L)$ $=\left\{w \in \Sigma^{*} \mid \exists v \in L: v \sqsubseteq w\right\}$ is the set of all continuations of language $L \subseteq \Sigma^{*}$.

A marking is a multiset over $P$; for example, $\left[p_{1}, 2 p_{2}\right]$ denotes a marking $m$ with $m\left(p_{1}\right)=1, m\left(p_{2}\right)=2$, and $m(p)=0$ for $p \in P \backslash\left\{p_{1}, p_{2}\right\}$. We define + and for the sum and the difference of two markings and $=,<,>, \leq, \geq$ for comparison of markings in the standard way. A marking is changed by firing a transition. A transition $t$ is enabled at a marking $m$, denoted by $m \stackrel{t}{\rightarrow}$, if $m \geq \bullet$. Then, $t$ can fire, reaching a marking $m^{\prime}$ (denoted by $m \stackrel{t}{\rightarrow} m^{\prime}$ ), where $m^{\prime}=m-\bullet+t^{\bullet}$.

The behavior of $N$ can be extended to sequences: $m_{1} \stackrel{t_{1}}{\longrightarrow} m_{2} \stackrel{t_{2}}{\longrightarrow} \ldots m_{k}$ is a run of $N$ if $m_{i} \stackrel{t_{i}}{\longrightarrow} m_{i+1}$, for all $0<i<k$. A marking $m^{\prime}$ is reachable from a marking $m$ if there exists a (possibly empty) run $m_{1} \stackrel{t_{1}}{\longrightarrow} \ldots \stackrel{t_{k-1}}{\longrightarrow} m_{k}$ with $m=m_{1}$ and $m^{\prime}=m_{k}$; for $w=t_{1} \ldots t_{k_{1}}$ we also write $m_{1} \stackrel{w}{\longrightarrow} m_{k}$. Marking $m^{\prime}$ is reachable if $m_{N}=m$. In the case of labeled nets, we lift runs to traces: If $m_{1} \stackrel{w}{\longrightarrow} m_{k}$ and $v$ is obtained from $w$ by replacing each transition by its label and removing all $\tau$ labels, we write $m_{1} \stackrel{v}{\Longrightarrow} m_{k}$ and call $v$ a trace whenever $m_{1}=m_{N}$. A marking $m$ is stable if it does not enable a $\tau$-labeled transition. The reachability graph $R G(N)$ of $N$ has the reachable markings as its nodes and a $t$-labeled edge from $m$ to $m^{\prime}$ whenever $m \stackrel{t}{\rightarrow} m^{\prime}$. In the case of a labeled net, each edge label $t$ is replaced by $l(t)$.

Finally, we introduce two properties of nets. A net $N$ is $b$-bounded for $b \in \mathbb{N}$ if $m(p) \leq b$ for every reachable marking $m$ and $p \in P ; N$ is bounded if it is $b$-bounded for some $b \in \mathbb{N}$. A reachable marking $m \notin \Omega$ of $N$ is a deadlock if no transition $t \in T$ of $N$ is enabled at $m$.

\subsection{Open Nets}

Like in 815, we model services as open nets [168], thereby restricting ourselves (like in [815]) to the communication protocol of a service. An open net extends a net by an interface. An interface consists of two disjoint sets of input and output places corresponding to asynchronous input and output channels. In the initial marking and the final markings, interface places are not marked. An input place has an empty preset, whereas an output place has an empty postset.

Definition 2 (open net). An open net $N$ is a tuple $\left(P, T, F, m_{N}, I, O, \Omega\right)$ with

- $\left(P \uplus I \uplus O, T, F, m_{N}, \Omega\right)$ is a net;

- for all $p \in I \uplus O: m_{N}(p)=0$ and for all $m \in \Omega: m(p)=0$;

- the set $I$ of input places satisfies ${ }^{\bullet} p=\emptyset$ for all $p \in I$; and

- the set $O$ of output places satisfies $p^{\bullet}=\emptyset$ for all $p \in O$.

Two open nets are interface equivalent if they have the same sets of input and output places. If $I=O=\emptyset$, then $N$ is a closed net. The net that results from removing the interface places and their adjacent arcs from $N$ is inner $(N)$. 


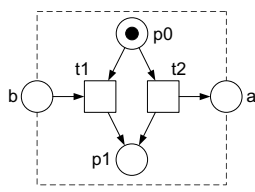

(a) $N_{1}$

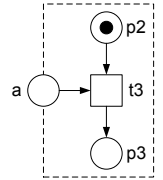

(b) $\mathrm{N}_{2}$

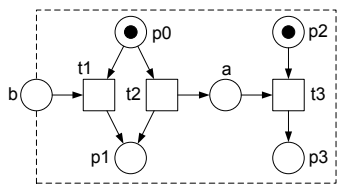

(c) $N_{1} \oplus N_{2}$

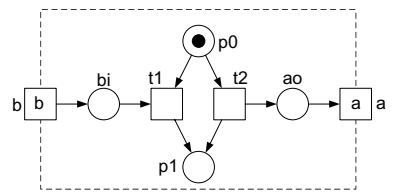

(d) $\operatorname{env}\left(N_{1}\right)$

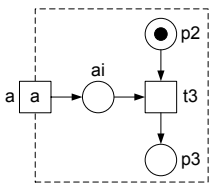

(e) $\operatorname{env}\left(N_{2}\right)$

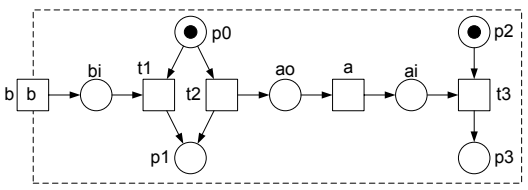

(f) $e n v\left(N_{1}\right) \Uparrow e n v\left(N_{2}\right)$

Fig. 1. Two open nets, their environments, and their composition

Graphically, we represent an open net like a net with a dashed frame around it. The interface places are depicted on the frame.

For the composition of open nets, we assume that the sets of transitions are pairwise disjoint and that no internal place of an open net is a place of any other open net. The interfaces intentionally overlap. We require that all communication is bilateral and directed; that is, every shared place $p$ has only one open net that sends into $p$ and one open net that receives from $p$. We refer to open nets that fulfill these properties as composable. We compose two composable open nets $N_{1}$ and $N_{2}$ by merging those interface places they have in common and turn these places into internal places.

Definition 3 (open net composition). Open nets $N_{1}$ and $N_{2}$ are composable if $\left(P_{1} \uplus T_{1} \uplus I_{1} \uplus O_{1}\right) \cap\left(P_{2} \uplus T_{2} \uplus I_{2} \uplus O_{2}\right)=\left(I_{1} \cap O_{2}\right) \uplus\left(I_{2} \cap O_{1}\right)$. The composition of two composable open nets $N_{1}$ and $N_{2}$ is the open net $N_{1} \oplus N_{2}=\left(P, T, F, m_{N}, I, O, \Omega\right)$, where

- P $=P_{1} \uplus P_{2} \uplus\left(I_{1} \cap O_{2}\right) \uplus\left(I_{2} \cap O_{1}\right) ; \quad T=T_{1} \uplus T_{2} ; \quad F=F_{1} \uplus F_{2} ;$

- $m_{N}=m_{N_{1}}+m_{N_{2}}$, extending $m_{N_{i}}$ with $m_{N_{i}}(p)=0 \forall p \in P_{3-i}, i=1,2$;

$-I=\left(I_{1} \uplus I_{2}\right) \backslash\left(O_{1} \uplus O_{2}\right) ; \quad O=\left(O_{1} \uplus O_{2}\right) \backslash\left(I_{1} \uplus I_{2}\right)$; and

$-\Omega=\left\{m_{1}+m_{2} \mid m_{1} \in \Omega_{1} \wedge m_{2} \in \Omega_{2}\right\}$.

Example 1. Figure 1 shows two composable open nets $N_{1}$ and $N_{2}$ and their composition $N_{1} \oplus N_{2}$. The composition is still an open net.

\subsection{Environments}

To give an open net $N$ a trace-based semantics, we consider its environment $e n v(N)$ similarly as in [16]. The net env $(N)$ can be constructed from $N$ by adding to each interface place $p \in I(p \in O)$ a $p$-labeled transition $p$ in $e n v(N)$ and renaming place $p$ by $p^{i}\left(p^{o}\right)$. This way, the asynchronous interface of $N$ is translated into a buffered synchronous interface described by the transition labels of $e n v(N)$. 
Definition 4 (open net environment). The environment of an open net $N$ is the labeled net $e n v(N)=\left(P \uplus P^{I} \uplus P^{O}, T \uplus I \uplus O, F^{\prime}, m_{N}, \Omega, I \uplus O, l^{\prime}\right)$, where

$$
\begin{aligned}
-P^{I}= & \left\{p^{i} \mid p \in I\right\} ; \quad P^{O}=\left\{p^{o} \mid p \in O\right\} ; \\
-F^{\prime}= & ((P \cup T) \times(T \cup P)) \cap F \\
\cup\left\{\left(p^{i}, t\right) \mid p \in I, t \in T,(p, t) \in F\right\} \cup\left\{\left(t, p^{o}\right) \mid p \in O, t \in T,(t, p) \in F\right\} & \cup\left\{\left(p^{o}, p\right) \mid p \in O\right\} \cup\left\{\left(p, p^{i}\right) \mid p \in I\right\} ; \text { and } \\
-l^{\prime}(t)= & \begin{cases}\tau, & t \in T \\
t, & t \in I \cup O .\end{cases}
\end{aligned}
$$

The language of $N$ is defined by $L(N)=\left\{w \in(I \uplus O)^{*} \mid m_{\text {env }(N)} \stackrel{w}{\Longrightarrow}\right\}$.

To compose environments of composable open nets, we define a parallel composition $\Uparrow$, where, for each action $a$ that the components have in common, each $a$-labeled transition of one component is synchronized with each $a$-labeled transition of the other; afterward $a$ is hidden. Because $a$ itself is the only $a$-labeled transition, the definition can be simplified compared to a more general setting, for instance in [16].

Definition 5 (parallel composition). Let env $\left(N_{1}\right)=\left(P_{1}, T_{1}, F_{1}, m_{N_{1}}, \Omega_{1}\right.$, $\left.\Sigma_{1}, l_{1}\right)$ and env $\left(N_{2}\right)=\left(P_{2}, T_{2}, F_{2}, m_{N_{2}}, \Omega_{2}, \Sigma_{2}, l_{2}\right)$ be the environments of composable open nets $N_{1}$ and $N_{2}$. The parallel composition of env $\left(N_{1}\right)$ and env $\left(N_{2}\right)$ is defined by the labeled net $\operatorname{env}\left(N_{1}\right) \Uparrow \operatorname{env}\left(N_{2}\right)=\left(P, T, F, m_{N}, \Omega, \Sigma, l\right)$, where

$-P=P_{1} \uplus P_{2} ; \quad T=T_{1} \cup T_{2} ;$

$-F=F_{1} \uplus F_{2} ; \quad m_{N}=m_{N_{1}}+m_{N_{2}} ;$

$-\Omega=\left\{m_{1}+m_{2} \mid m_{1} \in \Omega_{1} \wedge m_{2} \in \Omega_{2}\right\} ; \quad \Sigma=\left(\Sigma_{1} \cup \Sigma_{2}\right) \backslash\left(\Sigma_{1} \cap \Sigma_{2}\right)$; and

$-l(t)= \begin{cases}l_{1}(t), & t \in\left(T_{1} \backslash T_{2}\right) \\ l_{2}(t), & t \in\left(T_{2} \backslash T_{1}\right) \\ \tau, & \text { otherwise. }\end{cases}$

Example 2. Figure 1 depicts environment env $\left(N_{1}\right)$ and env $\left(N_{2}\right)$ of open nets $N_{1}$ and $N_{2}$ and their parallel composition env $\left(N_{1}\right) \Uparrow e n v\left(N_{2}\right) . L\left(N_{1}\right)=b^{*}+b^{*} a b^{*}$ and $L\left(N_{2}\right)=a^{*}$.

To describe the behavior of compositions, we define parallel compositions of words and languages; operator $\|$ synchronizes common actions, operator $\Uparrow$ also hides them. Observe that, in env $\left(N_{1}\right) \Uparrow \operatorname{env}\left(N_{2}\right)$, just common transitions are merged; operator $\|$ is needed to relate the respective transition sequences.

Definition 6. Given alphabets $\Sigma_{1}, \Sigma_{2}, \Sigma=\left(\Sigma_{1} \cup \Sigma_{2}\right) \backslash\left(\Sigma_{1} \cap \Sigma_{2}\right)$, words $w_{1} \in \Sigma_{1}^{*}$ and $w_{2} \in \Sigma_{2}^{*}$, and languages $L_{1} \subseteq \Sigma_{1}^{*}$ and $L_{2} \subseteq \Sigma_{2}^{*}$, we define

$-w_{1} \| w_{2}=\left\{w \in\left(\Sigma_{1} \cup \Sigma_{2}\right)^{*}|w|_{\Sigma_{1}}=\left.w_{1} \wedge w\right|_{\Sigma_{2}}=w_{2}\right\}$

$-w_{1} \Uparrow w_{2}=\left\{\left.w\right|_{\Sigma} \mid w \in w_{1} \| w_{2}\right\}$

- $L_{1} \| L_{2}=\bigcup\left\{w_{1} \| w_{2} \mid w_{1} \in L_{1} \wedge w_{2} \in L_{2}\right\}$; and

- $L_{1} \Uparrow L_{2}=\bigcup\left\{w_{1} \Uparrow w_{2} \mid w_{1} \in L_{1} \wedge w_{2} \in L_{2}\right\}$. 


\section{Trace Semantics for Deadlock Freedom}

This section considers the intuitive idea that the communication of a user with a service is successful if it only stops in case of successful termination; that is, the composition is in a final state. Such a user can also be seen as a controller of the service. When refining an open net Spec, understood as a service specification, to an implementing open net $I m p l$, we require that every satisfied user of Spec should also be satisfied with Impl. Our aim is to characterize this refinement relation without considering users or controllers explicitly, and to show that the relation is a precongruence for $\oplus$; that is, it supports compositional reasoning.

Definition 7 (controller, accordance). An open net $C$ is a controller of an open net $N$ if the composition $N \oplus C$ is a closed net without deadlocks.

Let Spec and Impl be interface equivalent open nets. Open net Impl accords with open net Spec, denoted by Impl $\sqsubseteq_{a c c} S p e c$, if every controller $C$ of $S p e c$ is also a controller of Impl.

Accordance has been defined in 15] for finite state services; that is, the composition of $N$ and a controller must be a bounded net. The unbounded case is interesting in its own right, and it is simpler in theory and prepares the approach for the bounded case.

\subsection{Stop-Dead Semantics}

Our new semantics of an open net $N$ considers two sets of traces for its environment env $(N)$. A stop-trace records a run of env $(N)$ that ends in a marking $m$ enabling transitions of $I$ only; it is a dead-trace if in addition $m$ is not a final marking.

Definition 8 (stop-dead (sd) semantics). Let $N$ be an open net. Marking $m$ of $e n v(N)$ is a stop except for inputs if $\forall t: m \stackrel{t}{\rightarrow}$ implies $t \in I$. Define

- $\operatorname{stop}(N)=\left\{w \mid m_{\operatorname{env}(N)} \stackrel{w}{\Longrightarrow} m\right.$ and $m$ is a stop except for inputs $\}$ and

$-\operatorname{dead}(N)=\left\{w \mid m_{e n v(N)} \stackrel{w}{\Longrightarrow} m \notin \Omega\right.$ and $m$ is a stop except for inputs $\}$.

With this semantics, we can characterize when a closed net has a deadlock.

Proposition 9. A closed net $N$ has a deadlock iff dead $(N)=\{\epsilon\}$.

We study now how the sd-semantics of two open nets can be combined to obtain the sd-semantics of their composition. For the rest of this section, we fix two composable open nets $N_{1}=\left(P_{1}, T_{1}, F_{1}, m_{N_{1}}, I_{1}, O_{1}, \Omega_{1}\right)$ and $N_{2}=$ $\left(P_{2}, T_{2}, F_{2}, m_{N_{2}}, I_{2}, O_{2}, \Omega_{2}\right)$, and we define $E=e n v\left(N_{1}\right) \Uparrow e n v\left(N_{2}\right)$. Note that env $\left(N_{1} \oplus N_{2}\right)$ and $E$ have the same places except for the $p \in\left(I_{1} \cap O_{2}\right) \cup\left(I_{2} \cap O_{1}\right)$ in $\operatorname{env}\left(N_{1} \oplus N_{2}\right)$ and the corresponding $p^{i}, p^{o}$ in $E$.

Theorem 10 (sd-semantics for open net composition). For composable open nets $N_{1}$ and $N_{2}$, we have

1. $L\left(N_{1} \oplus N_{2}\right)=L\left(N_{1}\right) \Uparrow L\left(N_{2}\right)$;

2. $\operatorname{stop}\left(N_{1} \oplus N_{2}\right)=\operatorname{stop}\left(N_{1}\right) \Uparrow \operatorname{stop}\left(N_{2}\right)$; and

3. $\operatorname{dead}\left(N_{1} \oplus N_{2}\right)=\left(\operatorname{dead}\left(N_{1}\right) \Uparrow \operatorname{stop}\left(N_{2}\right)\right) \cup\left(\operatorname{stop}\left(N_{1}\right) \Uparrow \operatorname{dead}\left(N_{2}\right)\right)$. 


\subsection{Coincidence with Accordance}

We define a refinement relation between open nets based on their sd-semantics. From Theorem 10, it is preserved under $\oplus$; that is, it is a precongruence.

Definition 11 (sd-refinement). For any two interface equivalent open nets Spec and Impl, Impl sd-refines Spec, denoted by Impl $\sqsubseteq_{\text {sd }}$ Spec, iff $\operatorname{dead}(\operatorname{Impl}) \subseteq$ $\operatorname{dead}($ Spec $) \wedge \operatorname{stop}(\operatorname{Impl}) \subseteq \operatorname{stop}($ Spec $)$.

Theorem 12 (precongruence). Relation $\sqsubseteq_{s d}$ is a precongruence for $\oplus$.

Next, we prove that the notion of accordance coincides with sd-refinement, and get a precongruence result as an easy corollary.

Theorem 13 (accordance and sd-refinement coincide). For interface equivalent open nets Spec and Impl, we have Impl $\sqsubseteq_{a c c}$ Spec $\Leftrightarrow I m p l \sqsubseteq s d$ Spec.

Corollary 14. Accordance is a precongruence for composition operator $\oplus$.

\section{Trace Semantics for Deadlock Freedom and Boundedness}

In this section, we restrict ourselves to finite state services - more precisely, a composition with a controller must be $b$-bounded for some $b \in \mathbb{N} \backslash\{0\}$. The motivation for this restriction is that we model the control flow of service compositions and assume a (finite) capacity of the message channels. To ensure boundedness in the composition, controllers must not send a message if there are already $b$ messages in the respective message channel, but they must also avoid bound violations on interior places. Technically, parameter $b$ enforces that the composition has a finite number of reachable states. Pragmatically, it could either represent a reasonable buffer size in the middleware - for example, the result of a static analysis of the communication behavior of a service - or be chosen sufficiently large.

Definition 15 (b-controllability). Let $b \in \mathbb{N} \backslash\{0\}$. An open net $C b$-controls an open net $N$ if the composition $N \oplus C$ is closed, deadlock-free, and $b$-bounded.

Different from [8], we also prescribe bound $b$ for the interior of services and controllers. Instead, only boundedness with possibly varying bounds is required in [8]. That this requirement is weaker is of little relevance], but it can create problems, because interface places turn into interior places of a composition. More importantly, boundedness is required in [8] for the inner of services and controllers on their own - that is, with unlimited input tokens in particular. We are more general, because we allow services (and users) that have an unbounded

\footnotetext{
${ }^{1}$ In principle, one can determine the state space of such a bounded open net and transform it into an automaton (such automata are studied in [8], for example) and, thus, into an open net which has even a 1-bounded inner.
} 
inner; we only require that they are $b$-bounded in suitable contexts. Thus, we are more optimistic in the sense of [3], because we assume that the controller will steer the service away from bound violations.

We lift accordance for open nets to $b$-accordance by comparing $b$-controllers only. In [15, 1-accordance has been defined for controllers as defined in 8].

Definition 16 (b-accordance). For interface equivalent open nets Spec and Impl, Impl b-accords with Spec, denoted by Impl $\sqsubseteq_{a c c}^{b} S p e c$, if $\forall C: C$ b-controls Spec implies $C$ b-controls $I m p l$.

In the remainder of this section, we extend the sd-semantics of open nets taking into account the bound. With the new semantics, we define a refinement relation between open nets and prove that it coincides with $b$-accordance.

\subsection{Bounded Stop-Dead Semantics}

The idea is to specify a set $b d_{b}(N)$ containing all traces of $e n v(N)$ that lead to a marking violating bound $b$. We refer to such a trace $v$ as a bound-violator. As we want to forbid such a trace, a continuation $w$ of $v$ is automatically forbidden as well; thus, it is irrelevant whether $w$ is a bound-violator or can be performed at all. Technically, this abstraction from continuations is achieved by including all continuations of bound-violators in $b d_{b}(N)$. For the same reason, $b d_{b}(N)$ is contained in all other components of our semantics. This is similar to the treatment of divergence traces in failures semantics [2] and also of $U(N)$ in [16. Def. 3.3.2] — for a setting with synchronous communication in both cases.

Definition 17 (bounded stop-dead (bsd-) semantics). Let $b \in \mathbb{N} \backslash\{0\}$. For an open net $N=\left(P, T, F, m_{N}, I, O, \Omega\right)$ with environment $e n v(N)$, we call a trace $v$ a bound $d_{b}$-violator of $N$ if $m_{e n v(N)} \stackrel{v}{\Longrightarrow} m \wedge \exists p \in P \cup I \cup O: m(p)>b$.

$-b d_{b}(N)=\left\{w \in(I \cup O)^{*} \mid \exists v \sqsubseteq w: v\right.$ is a bound $_{b}$-violator $\}$,

- $L_{b}(N)=L(N) \cup b d_{b}(N)$,

$-\operatorname{stop}_{b}(N)=\operatorname{stop}(N) \cup b d_{b}(N)$, and

$-\operatorname{dead}_{b}(N)=\operatorname{dead}(N) \cup b d_{b}(N)$.

Just as $\operatorname{stop}\left(N_{1}\right)$ is needed to determine $\operatorname{dead}\left(N_{1} \oplus N_{2}\right), L_{b}\left(N_{1}\right)$ will be needed to determine $b d_{b}\left(N_{1} \oplus N_{2}\right)$.

We characterize the bsd-semantics of the composition of two open nets based on their bsd-semantics, enforcing continuation closure of $b d_{b}\left(N_{1} \oplus N_{2}\right)$ explicitly.

Theorem 18 (bsd-semantics for open net composition). For composable open nets $N_{1}$ and $N_{2}$ we have

1. $b d_{b}\left(N_{1} \oplus N_{2}\right)=\operatorname{cont}\left(\left(b d_{b}\left(N_{1}\right) \Uparrow L_{b}\left(N_{2}\right)\right) \cup\left(L_{b}\left(N_{1}\right) \Uparrow b d_{b}\left(N_{2}\right)\right)\right)$

2. $L_{b}\left(N_{1} \oplus N_{2}\right)=\left(L_{b}\left(N_{1}\right) \Uparrow L_{b}\left(N_{2}\right)\right) \cup b d_{b}\left(N_{1} \oplus N_{2}\right)$

3. $\operatorname{stop}_{b}\left(N_{1} \oplus N_{2}\right)=\left(\operatorname{stop}_{b}\left(N_{1}\right) \Uparrow \operatorname{stop}_{b}\left(N_{2}\right)\right) \cup b d_{b}\left(N_{1} \oplus N_{2}\right)$

4. $\operatorname{dead}_{b}\left(N_{1} \oplus N_{2}\right)$

$$
=\left(\operatorname{stop}_{b}\left(N_{1}\right) \Uparrow \operatorname{dead}_{b}\left(N_{2}\right)\right) \cup\left(\operatorname{dead}_{b}\left(N_{1}\right) \Uparrow \operatorname{stop}_{b}\left(N_{2}\right)\right) \cup b d_{b}\left(N_{1} \oplus N_{2}\right)
$$




\subsection{Coincidence with $b$-Accordance and Nonredundancy}

Analogously to sd-refinement, we define bsd-refinement and conclude from Theorem 18(1) to (4) that it is preserved under the composition operator $\oplus$.

Definition 19 (bsd-refinement). For interface equivalent open nets Spec and Impl and a bound $b \in \mathbb{N} \backslash\{0\}$, Impl bsd $d_{b}$-refines Spec, denoted by Impl $\sqsubseteq_{b s d, b}$ Spec, if $L_{b}(\operatorname{Impl}) \subseteq L_{b}($ Spec $) \wedge \operatorname{dead}_{b}(\operatorname{Impl}) \subseteq \operatorname{dead}_{b}(\operatorname{Spec}) \wedge \operatorname{stop}_{b}(\operatorname{Impl}) \subseteq$ $\operatorname{stop}_{b}($ Spec $) \wedge b d_{b}(\operatorname{Impl}) \subseteq b d_{b}($ Spec $)$.

Theorem 20 (precongruence). For each $b \in \mathbb{N} \backslash\{0\}$, bs $d_{b}$-refinement is a precongruence for composition operator $\oplus$.

Each refinement relation $b$-accordance (see Definition [16) coincides with bsd $_{b^{-}}$ refinement, which implies a novel precongruence result as a corollary. The main point for the latter result is that we have a more uniform approach compared to [8, where in Definition [15 the interface places of $N$ and $C$ play a special role in $N \oplus C$ although they are ordinary places of this closed net. In fact, $b$-accordance, as introduced in [15, is no precongruence for our composition operator $\oplus$. Corollary 22 could also be proved directly by a standard argument, as presented in a very general setting in [13].

Theorem 21 ( $\sqsubseteq_{a c c, b}$ and $\sqsubseteq_{b s d, b}$ coincide). For interface equivalent open nets Spec and Impl and $a$ bound $b \in \mathbb{N} \backslash\{0\}$ holds Impl $\sqsubseteq_{a c c, b}$ Spec $\Leftrightarrow \operatorname{Impl} \sqsubseteq_{b s d, b}$ Spec.

Corollary 22. For each $b \in \mathbb{N} \backslash\{0\}$, b-accordance is a precongruence for $\oplus$.

As the bsd-semantics consists of four overlapping components, the question arises whether each of them is really needed. In particular, one might suspect that the set $L_{b}$ can be obtained from the other three sets by taking prefixes. To answer this question, one can construct examples showing that none of the components can be deduced from the others.

\subsection{Full Abstractness}

We can present the results of Sect. 4.2 also as a full abstractness result [11; that is, $b$-accordance is the coarsest precongruence for open nets w.r.t. a suitable basic relation $\sqsubseteq_{b}$ and composition operator $\oplus$. It is somewhat unusual that this basic relation is only defined for closed nets: Impl $\sqsubseteq_{b}$ Spec says that Impl must represent a 'good' communication if Spec does.

Definition 23 (basic relation). For closed open nets Spec and Impl and a bound $b \in \mathbb{N} \backslash\{0\}$, define the basic relation Impl $\sqsubseteq_{b}$ Spec iff whenever Spec cannot deadlock and does not violate bound $b$ so does Impl.

Relation $\sqsubseteq_{b}^{c}$ is the coarsest precongruence for $\oplus$ such that for all closed Impl and Spec: Impl $\sqsubseteq_{b}^{c}$ Spec implies Impl $\sqsubseteq_{b}$ Spec.

Theorem 24 ( $\sqsubseteq_{a c c, b}$ is fully abstract). For interface equivalent open nets Spec and Impl and $a$ bound $b \in \mathbb{N} \backslash\{0\}$ holds Impl $\sqsubseteq_{a c c, b}$ Spec $\Leftrightarrow \operatorname{Impl} \sqsubseteq_{b}^{c}$ Spec. 


\subsection{Deciding bsd-Refinement}

To decide bsd-refinement between two open nets, four language inclusions must be checked. We discuss a decision procedure for bsd-refinement, also to prepare the comparison to the standard approach for deciding $b$-accordance (see [15]), which is based on operating guidelines [8].

For deciding the four language inclusions, we build one automaton with four types of final states. To do this, we construct the reachability graph of the labeled net $\operatorname{env}(N)$ under consideration, but stop the construction whenever we reach a marking $m$ that violates the bound $b$. As any bounded net has only finitely many reachable markings, this guarantees finiteness of our construction. Each such marking $m$ gets a loop for each visible label from $I \cup O$. The initial state of the automaton is the initial marking $m_{e n v(N)}$.

This way, the automaton has $b d_{b}(N)$ as language if we designate each $m$ that violates $b$ as final state. If we additionally take those markings $m$ as final states that are stops except for inputs and not in the set $\Omega$ of final markings, then the language is $\operatorname{dead}_{b}(N)$. If we add all markings $m$ as final states that are stops except for inputs, then the language is $\operatorname{stop}_{b}(N)$. Finally, letting all states be final, the language is $L_{b}(N)$.

If we label the final states added in the four stages with $0,1,2,3$, then, for each state, we need two additional bits for encoding the four languages. We use

- 0 to describe the language $b d_{b}(N)$;

- 0 and 1 to describe the language $\operatorname{dead}_{b}(N)$;

$-0,1$, and 2 to describe the language $\operatorname{stop}_{b}(N)$; and

- $0,1,2$, and 3 to describe the language $L_{b}(N)$.

For checking language inclusion, one makes at least the automaton for the larger language deterministic (by constructing the powerset automaton) and constructs the (unique minimal) simulation 12 between the two automata. Practically, this powerset construction often leads to smaller automata. In our case, it suffices to apply this construction once - although we have four languages. A similar construction is used when building a lexer, for instance. Here, we refer to a version of the powerset construction that also removes $\tau$-transitions - that is, $\epsilon$-transitions according to automata theory.

Each set of states, as state of the powerset automaton, is a node and is labeled with the minimum label of its states. As a further simplification, one can identify all nodes with label 0 into one node $U$. This identification is integrated into the powerset construction. We refer to the resulting automaton as $B S D_{b}(N)$.

To decide bsd-refinement of two open nets $S p e c$ and $I m p l$, we calculate the minimal simulation $\varrho$ of $B S D_{b}(I m p l)$ by $B S D_{b}(S p e c)$ and check the labels of related states as stated next; one could also consider the minimal simulation of the modified reachability graph of $I m p l$, as described previously, by $B S D_{b}(S p e c)$.

Theorem 25 (deciding bsd-refinement). For interface equivalent open nets Spec and Impl and $a$ bound $b \in \mathbb{N} \backslash\{0\}$, we have Impl $\sqsubseteq_{b s d, b}$ Spec iff the minimal simulation of $B S D_{b}(\mathrm{Impl})$ by $B S D_{b}($ Spec $)$ exists and relates a node of $B S D_{b}(\operatorname{Impl})$ with label $i$ only to nodes of $B S D_{b}($ Spec $)$ with label $j \leq i$. 


\section{Comparing bsd-Semantics and Operating Guidelines}

We compare the bsd-semantics of Definition 17 with the notion of an operating guideline 8 for open nets $N$. An operating guideline $O G_{b}(N)$ of a service $N$ describes how a user should successfully communicate with $N$; technically, it characterizes the possibly infinite set of $b$-controllers of $N$ in a finite manner. Because a $b$-controller of $N$ provides suitable inputs for $N$ and accepts its outputs, $O G_{b}(N)$ is similar to $B S D_{b}(N)$ but with inputs and outputs interchanged.

The operating guidelines of two open nets $S p e c$ and $I m p l$ can be used to decide that Impl b-accords with Spec 15. Thus, $O G_{b}(N)$ and $B S D_{b}(N)$ should have the same semantic content. As their details are rather different, we clarify their relation by showing how to translate $O G_{b}(N)$ and $B S D_{b}(N)$ into each other. These translations do not make use of the states contained in the nodes, but the correctness proof for the translations does; for example, the node labels are defined on the basis of theses states.

Operating guidelines have been defined only for services $N$ with $b$-bounded interface for which inner $(N)$ is bounded. As these two bounds are not necessarily the same, we restrict the translation to services $N$ where $\operatorname{inner}(N)$ is $b$-bounded.

\subsection{Deriving an Operating Guideline from the bsd-Semantics}

Both $O G_{b}(N)$ and $B S D_{b}(N)$ are finite automata that can be distinguished by their graph structure and annotations.

Graph structure. There are two differences in the graph structure of $O G_{b}(N)$ and $B S D_{b}(N)$. First, a $b$-controller may be able to accept inputs that $N$ will never send. As a consequence, $O G_{b}(N)$ contains an additional node, the empty node (which does not contain any state). For each output $x \in O$ of $N$ and each node without an outgoing $x$-labeled edge in $B S D_{b}(N)$, there is a respective edge leading to the empty node, and this node has loops for all possible visible actions from $I \cup O$. Because $B S D_{b}(N)$ represents the behavior of $N$ rather than the behavior of its $b$-controllers, the empty node does not exist in $B S D_{b}(N)$. To ease the presentation, we ignore the empty node in the following.

Second, node $U$, which identifies all nodes with label 0 in $B S D_{b}(N)$, is not present in $O G_{b}(N)$. Because $O G_{b}(N)$ characterizes all $b$-controllers, possible bound violations will not happen and, hence, do not need to be stored. It is easy to omit $U$ from $B S D_{b}(N)$, as it is identified by label 0 . Vice versa, it is also possible to add $U$ to $O G_{b}(N)$ by adding, for each input $a \in I$ of $N$ to each node of $O G_{b}(N)$ without an outgoing $a$-labeled edge, such an edge leading to $U$. In addition, node $U$ has like the empty node loops for all visible actions. Note that the empty node and node $U$ are characterized in their respective automaton by having a loop for a respective output action, leading to behavior that certainly violates any bound $b$.

Annotations. Instead of the remaining node labels $1,2,3$ in $B S D_{b}(N)$, each node $Q$ of $O G_{b}(N)$ is annotated with a Boolean formula $\phi(Q)$. The propositional 
atoms of $\phi$ are $I \cup O \cup\{$ final $\}$. A $b$-controller cannot know which state $q$ of a node $Q$ net env $(N)$ might be in, but it has to avoid a deadlock and a bound violation in any case; the formula $\phi(Q)$ describes how to do this. Nonstable states have an internal transition and, thus, are not deadlocks; all internal transitions remain in the same node. As a consequence, $\phi(Q)$ is a conjunction indexed by all stable states $q \in Q$. Every conjunct is a disjunction of the following propositional atoms: final if $q$ is a final state, $a \in I$ if $Q \stackrel{a}{\longrightarrow}$, and $x \in O_{N}$ if $q \stackrel{x}{\longrightarrow}$. Hence, the formulae are in conjunctive normal form (CNF) without negation.

To construct $\phi(Q)$ from $B S D_{b}(N)$ (up to equivalence), we need a procedure to which we refer to as 1,2-DFS. This procedure is something like a depth-first search through the automaton starting from node $Q$. It considers only $x$-labeled edges with $x \in O_{N}$ and backtracks if (and only if) there is no further such edge or when encountering a 1,2-node (i. e., a node labeled 1 or 2); that is, visited states are not marked as visited in this procedure. When a 1,2-node is encountered, the path in the stack is called a maximal path constructed during 1,2-DFS. One can see that 1,2-DFS never encounters node $U$, because edges leading to $U$ are input edges of $N$. Also note that 1,2-DFS cannot run into a cycle; if $Q \stackrel{x}{\longrightarrow} Q^{\prime}$, then $Q^{\prime}$ consists of all $q^{\prime}$ such that $\exists q \in Q: q \stackrel{x}{\longrightarrow} q^{\prime}$; in each case, the markings $q$ and $q^{\prime}$ coincide except that $q^{\prime}\left(x^{o}\right)=q\left(x^{o}\right)-1$.

As the formula $\phi(Q)$ depends on the stable states $q \in Q$, we first deduce whether node $Q$ contains any stable state.

Lemma 26. A node $Q$ of $B S D_{b}(N)$ contains a stable state iff 1,2-DFS starting from $Q$ encounters a 1,2-node.

So far, we considered nodes $Q$ that contain a stable state. If node $Q$ does not contain a stable state, then $\phi(Q)$ is the empty conjunction which is equal to true. In this case, node $Q$ has label 3 .

Lemma 27. $Q$ does not contain a stable state iff $\phi(Q)$ is the empty conjunction iff $\phi(Q)$ is true. If these three statements hold, then $Q$ has label 3.

For nodes without a stable state, we know by Lemmata 26 and 27 how to translate label and formula into each other, and we can assume for the following that $\phi(Q)$ has a conjunct. $Q$ can have label 1, 2, or 3; the next two lemmata characterize the nodes with label 1 and 2 , respectively.

By definition of formula $\phi(Q)$, all conjuncts have those inputs $a$ of $N$ as literals such that there is an $a$-labeled edge leaving $Q$. We factor out the disjunction of these inputs from $\phi(Q)$, and it remains to construct the remaining disjunct which is a $\mathrm{CNF} C$. If this remaining $C N F C$ has a conjunct false, then $C$ is equivalent to false and we can reconstruct $\phi(Q)$ from the edges leaving $Q$. In this case, $Q$ is labeled 1 .

Lemma 28. The remaining $C N F C$ of some node $Q$ has a conjunct false iff $Q$ is labeled 1.

We are left with considering nodes that are not labeled 1 and whose remaining $\mathrm{CNF} C$ is not equivalent to false. For such nodes, we determine whether there is some conjunct containing a literal final. 
Lemma 29. Assuming that node $Q$ is not labeled 1, the remaining CNF $C$ of $Q$ has a conjunct containing final iff $Q$ is labeled 2. Then the respective conjunct equals the literal final.

Finally, we determine the remaining conjuncts for which it suffices to find at least the minimal ones regarding each conjunct as the set of its literals. These are sets of edge labels of the maximal paths being constructed during the 1,2-DFS.

Lemma 30. Let $C$ be the remaining $C N F$ of a node $Q$. The sets of edge labels of the maximal paths being constructed during 1,2-DFS are conjuncts of $C$. Each conjunct of $C$ that is not false or equal to final contains the set of edge labels of some maximal path constructed during 1,2-DFS.

\subsection{Deriving bsd-Semantics from Operating Guidelines}

The previous results also show how to derive $B S D_{b}(N)$ from $O G_{b}(N)$; we collect the respective observations. First, we add the 0 -labeled node $U$, as previously described, and we remove the empty node. For the other nodes, we run through our preceding considerations for constructing $\phi$.

If $\phi(Q)$ is true, then there is no stable state $q \in Q$ and the label of $Q$ is, therefore, 3 (see Lemma 27).

Otherwise, if $\phi(Q)$ is equivalent to the disjunction of those inputs $a$ of $N$ such that there is an $a$-labeled edge leaving $Q$, then the remaining CNF is false and the label of $Q$ is 1 (see Lemma 28).

Otherwise, the remaining $\mathrm{CNF} C$ is not equal to false and we have to check whether final is a conjunct of $C$. By Lemma 29, this is the case if and only if the label of $Q$ is 2. (To check semantically whether final is a conjunct without relying on the precise form of $\phi$ of $C$, we assign true to all outputs of $N$ and false to all inputs and to final.) Then $\phi(Q)$ evaluates to false if and only if final is a conjunct. To all remaining nodes we assign label 3 .

\subsection{Accordance Check with Operating Guidelines}

For open nets Spec and Impl, with operating guidelines $O G_{b}(S p e c)$ and $O G_{b}(\operatorname{Impl})$, we have that $\operatorname{Impl} \sqsubseteq_{a c c, b} S p e c$ if and only if there exists a minimal simulation $\varrho$ of $O G_{b}($ Spec $)$ by $O G_{b}(\operatorname{Impl})$ and, for each pair of nodes $\left(Q, Q^{\prime}\right) \in \varrho$, $\phi(Q)$ implies $\phi\left(Q^{\prime}\right)$ is a tautology [15].

Interestingly, this check shows that the simulation relation is the other way around compared to the bsd-setting. Checking $b$-accordance involves repeated checks of implications between the annotations, whereas in the bsd-setting (see Theorem 25), we simply compare numbers $0,1,2,3$ in constant time.

\section{Conclusions}

We presented a novel semantics for open nets assuming the absence of deadlocks as a minimal correctness criterion. The semantics consists of four sets of traces. 
The set $b d_{b}(N)$ collects catastrophic traces due to bound violation; these traces modify all components just as divergence traces in failures semantics [2]. The sets $\operatorname{stop}_{b}(N)$ and $\operatorname{dead}_{b}(N)$ are successful and unsuccessful completed traces; because we are in an asynchronous setting, they can be continued with inputs; that is, they are quiescent as in I/O automata [10]. The fourth component, $L_{b}$, is just the language of the net $N$, comprising all traces.

We proved that our semantics can be translated back and forth into operating guidelines, and we derived the bsd-refinement relation from it, which coincides with (a slight modification of the) accordance relation. In addition, we proved bsd-refinement to be a fully abstract precongruence.

The bsd-semantics is related to the recently proposed notion of a reduced operating guideline 9 . The idea is to distinguish three disjoint subsets of nodes in an operating guideline such that the Boolean formulae can be derived from this information. The argumentation that leads to this representation has similarities to our arguments for translating $O G_{b}(N)$ into $B S D_{b}(N)$. However, in 9 responsiveness is considered: in addition to deadlock freedom, a $b$-controller in [9] also has to guarantee that in each state of the composition either a final state can be reached or a message is exchanged.

Different precongruences for asynchronously communicating processes have been studied. The introduced bsd-refinement is closely related to previous work of Vogler [16], closest to P-deadlock refinement. As a difference, in the setting of Vogler [16], the interface is not separated into input and output places, interface places may be unbounded (like in the sd-semantics), and the notion of a deadlock ignores $\tau$-loops: a marking $m$ is a deadlock if $m \stackrel{w}{\Longrightarrow}$ implies $w=\epsilon$. This leads to a much more complicated characterization.

Testing equivalence for asynchronous CCS is considered in [1, but it is an asymmetric notion focusing on the controller (i.e., the test) and not on the service; in contrast, the defining condition in Definitions 7 and [15] is symmetric in $C$ and $N$. Avoidance of deadlocks is not so essential, and in fact $\tau$-loops are not helpful or even a catastrophe; a process may receive the messages it has sent.

Stuck-free conformance [6] is a precongruence that excludes deadlocks. In contrast to bsd-refinement, it is based on a variation of failures semantics rather than traces. In [6], it has been proved that stable failures refinement [2] does not imply stuck-free conformance. In contrast, it has already been argued in a shared-variable setting in [4, that refusal sets are not needed when working with asynchronous communication.

In the area of SOC, the subcontract preorder [7] is an asymmetric notion also focusing on the tests. It is based on synchronous communication and only requires that the controller never gets stuck. In [7], it has been proved that the subcontract preorder coincides with must-testing [5] - that is, with stable failures refinement (for finitely branching processes without divergences).

Our goal was to provide a trace-based view on operating guidelines. In ongoing work, we investigate how the bsd-semantics of a service $S$ can be used to check whether a service is a controller of $S$. In addition, we want to gain a better understanding which transformations bsd-refine a service, because these 
transformations allow us to construct replaceable services. We will further study how our semantics needs to be adjusted if we consider responsiveness [9] rather than deadlock freedom. Here, we are particularly interested in the relation of the resulting semantics and the notion of a reduced operating guideline [9].

\section{References}

1. Boreale, M., De Nicola, R., Pugliese, R.: Trace and testing equivalence on asynchronous processes. Inf. Comput. 172(2), 139-164 (2002)

2. Brookes, S.D., Hoare, C.A.R., Roscoe, A.W.: A theory of communicating sequential processes. J. ACM 31(3), 560-599 (1984)

3. de Alfaro, L., Henzinger, T.A.: Interface automata. In: ESEC / SIGSOFT FSE 2001, pp. 109-120 (2001)

4. de Boer, F.S., Kok, J.N., Palamidessi, C., Rutten, J.J.M.M.: The failure of failures in a paradigm for asynchronous communication. In: Groote, J.F., Baeten, J.C.M. (eds.) CONCUR 1991. LNCS, vol. 527, pp. 111-126. Springer, Heidelberg (1991)

5. De Nicola, R., Hennessy, M.: Testing equivalences for processes. Theor. Comput. Sci. 34, 83-133 (1984)

6. Fournet, C., Hoare, T., Rajamani, S.K., Rehof, J.: Stuck-Free Conformance. In: Alur, R., Peled, D.A. (eds.) CAV 2004. LNCS, vol. 3114, pp. 242-254. Springer, Heidelberg (2004)

7. Laneve, C., Padovani, L.: The must preorder revisited. In: Caires, L., Li, L. (eds.) CONCUR 2007. LNCS, vol. 4703, pp. 212-225. Springer, Heidelberg (2007)

8. Lohmann, N., Massuthe, P., Wolf, K.: Operating guidelines for finite-state services. In: Kleijn, J., Yakovlev, A. (eds.) ICATPN 2007. LNCS, vol. 4546, pp. 321-341. Springer, Heidelberg (2007)

9. Lohmann, N., Wolf, K.: Compact representations and efficient algorithms for operating guidelines. Fundam. Inform. (2010) (accepted for publication in January 2010)

10. Lynch, N.: Distributed Algorithms. Morgan Kaufmann, San Francisco (1996)

11. Milner, R.: Fully abstract models of typed lambda-calculi. Theor. Comput. Sci. 4(1), 1-22 (1977)

12. Milner, R.: Communication and Concurrency. Prentice-Hall, Inc., Englewood Cliffs (1989)

13. Mooij, A.J., Voorhoeve, M.: Proof techniques for adapter generation. In: Bruni, R., Wolf, K. (eds.) WS-FM 2008. LNCS, vol. 5387, pp. 207-223. Springer, Heidelberg (2009)

14. Papazoglou, M.P.: Web Services: Principles and Technology. Pearson, London (2007)

15. Stahl, C., Massuthe, P., Bretschneider, J.: Deciding substitutability of services with operating guidelines. In: Jensen, K., van der Aalst, W.M.P. (eds.) Transactions on Petri Nets and Other Models of Concurrency II. LNCS, vol. 5460, pp. 172-191. Springer, Heidelberg (2009)

16. Vogler, W.: Modular Construction and Partial Order Semantics of Petri Nets. LNCS, vol. 625. Springer, Heidelberg (1992) 